\title{
Latitudinal and cold-tolerance variation associate with DNA repeat-number variation in the hsr-omega RNA gene of Drosophila melanogaster
}

\author{
JE Collinge ${ }^{1}$, AR Anderson ${ }^{2}$, AR Weeks ${ }^{3}$, TK Johnson and SW McKechnie \\ Centre for Environmental Stress and Adaptation Research (CESAR), School of Biological Sciences, Monash University, Melbourne, \\ Victoria, Australia
}

\begin{abstract}
An 8-bp deletion in the hsr-omega heat-stress gene of Drosophila melanogaster has previously been associated with latitude, and with heat tolerance that decreases with latitude. Here we report a second polymorphic site, at the $3^{\prime}$-end of $h s r-$ omega, at which multiple alleles segregate in natural populations for copy number of a $\sim 280 \mathrm{bp}$ tandem repeat. On each of 3 consecutive years (2000, 2001 and 2002) among populations sampled along the Australian eastern coast, repeat number was negatively associated with latitude. Neither altitudinal association was detected in 2002 when five high-altitude sites were included, nor was a robust association detected with local temperature or rainfall measures. Although in a large number of family lines, derived from a population located centrally in the latitudinal transect, no association between hsr-omega repeat number and heat tolerance occurred, a negative association of
\end{abstract}

repeat number with cold tolerance was detected. As cold tolerance also exhibits latitudinal clines we examined a set of cold-tolerant populations derived by selection and found both reduced repeat number and low constitutive levels of the omega- $n$ repeat-bearing transcript. In a sample from the central population, linkage disequilibrium was measured between repeat number and linked markers that also cline latitudinally. However, such disequilibrium could not account for the cline in repeat number or tolerance associations. Finally, during adult recovery from cold exposure a large increase occurred in tissue levels of the omega- $c$ transcript. Together these data suggest that a latitudinal cline in hsr-omega repeat number influences cold-tolerance variation in this species.

Heredity (2008) 101, 260-270; doi:10.1038/hdy.2008.57; published online 18 June 2008

Keywords: hsr-Omega repeats; latitudinal variation; Drosophila melanogaster, cold tolerance

\section{Introduction}

Physiological and genetic mechanisms that underpin thermal tolerance variation among species and among ecotypes of the same species have long been a topic of interest (Cossins and Bowler, 1987; Hoffmann and Parsons, 1991; Zatsepina et al., 2000) especially now that we have a heightened awareness of the threats to animal and plant populations under global warming (Lovejoy and Hanna, 2006). Drosophila species have been of considerable focus in attempts to elucidate such mechanisms because, apart from the obvious benefits of their experimental and genomic tractability, there are numerous examples of closely related species with quite different thermal tolerance attributes (Hoffmann et al.,

Correspondence: Professor SW McKechnie, Centre for Environmental Stress and Adaptation Research (CESAR), School of Biological Sciences, Monash University, Melbourne, Victoria 3800, Australia.

E-mail: stephen.mckechnie@sci.monash.edu.au

${ }^{1}$ Current address: Molecular Medicine Division, The Walter \& Eliza Hall Institute of Medical Research, 4 Research Avenue, La Trobe RED Park, Bundoora, Victoria 3086, Australia.

${ }^{2}$ Current address: CSIRO, Division of Entomology, GPO Box 1700, Canberra, Australian Capital Territory 2601, Australia.

${ }^{3}$ Current address: CESAR, Department of Genetics, University of Melbourne, Parkville, Victoria 3010, Australia.

Received 20 December 2007; revised 19 May 2008; accepted 23 May 2008; published online 18 June 2008
2003) and of intra-specific ecotype variation where strains from warm tropical regions at low latitudes are more heat tolerant and/or more cold sensitive than those from cool temperate higher latitudes (Kimura et al., 1994; Guerra et al., 1997; Hoffmann et al., 2002). Heritable, climatically-associated variation in stress resistance traits provide the opportunity to investigate and understand the potential of and limits to the processes of adaptation to thermal extremes.

A large number of genes have been identified that are helping to elucidate cellular and physiological mechanisms that protect from heat stress (Hoffmann et al., 2003; Sørensen et al., 2005). Multiple processes are clearly involved and these need to be identified and understood. Of the candidate genes that have been the subject of more intensive investigation, the best characterized, especially in Drosophila, is the hsp70 gene (see Bettencourt et al., 2002 and Gong and Golic, 2006, and references therein). Although it is generally recognized that $h s p 70$ is an essential component of the cellular heat-stress response, and that in general high levels of expression of $h s p 70$ are more protective, some measures of heat resistance show a negative association with expression of $h s p 70$ (Zatsepina et al., 2001; and see discussion in Hoffmann et al., 2003 and Chen et al., 2008). Other reports implicate the importance of different heat-shock protein genes (McColl et al., 1996) and novel genes (Ekengren and Hulmark, 2001) for thermal tolerance variation. 
In Drosophila melanogaster one candidate gene that has been subject to thorough investigation at the molecular and cellular level is hsr-omega (Pardue et al., 1990). This gene does not produce a protein product. It produces two main RNA transcripts both of which are constitutively expressed in most adult tissue types, one located in the cytoplasm, omega-c, and one in the nucleus, omega- $n$. hsr-Omega has long been recognized as one of the heatstress genes as it is upregulated by a mild heat shock (similar to the heat-shock protein genes), and because overlapping deletion mutants of hsr-omega are thermosensitive (Pardue et al., 1990). Also, hsr-omega shares with the other heat-shock genes isolated from natural populations an abundance of $P$-element inserts in the promoter region (Walser et al., 2006) - inserts that may influence heat-shock gene expression and thermal adaptive potential (Chen et al., 2008).

Further, in parallel with clines in thermal tolerance along a latitudinal gradient on the eastern coast of Australia, an 8-bp indel polymorphism in the hsr-omega gene (the hsr-omega-L/S indel polymorphism; Figure 1) shows a strong frequency cline that has been associated with average maximum temperatures of the hottest month (Anderson et al., 2003). The shorter allele (hsromega-S), characterized by the 8-bp deletion in the first exon ( 80 bp upstream of the intron $5^{\prime}$ splice site; Figure 1; McKechnie et al., 1998), occurs at high frequency in Australian tropical populations, and is strongly and negatively associated with latitude (Anderson et al., 2003). Furthermore, both hsr-omega-S allele and hsr-omega transcript levels have been associated with heat-stress resistance in selected lines (McColl et al., 1996; McKechnie et al., 1998).

Various measures of heat resistance have been used in recent studies, some of which have found associations with hsr-omega-L/S variation and some of which have not (Weeks et al., 2002; Norry et al., 2004; Morgan and Mackay, 2006; Rako et al., 2007). One of the confounding factors in these association studies is the linkage disequilibrium of hsr-omega with breakpoints of the common cosmopolitan inversion In(3R)Payne (Kennington et al., 2006). The hsr-omega gene is cytologically located within this major cosmopolitan inversion, and the hsr-omega-S allele is strongly associated with its presence (Anderson et al., 2005a). Generally, inversions are known to maintain certain allele combinations across loci by suppressed recombination, thus in this instance any genotype/resistance association only provides weak evidence of a causal role for hsr-omega.

What we know about the cellular biology of the hsromega transcripts however is consistent with it having a role in the heat-stress response that has been well characterized in Drosophila (Lindquist, 1980). Although the heat-stress response involves many genes (Sørensen et al., 2005), a major component is the upregulation of heat-shock protein synthesis and downregulation of normal protein synthesis. Under heat stress, the hsromega transcripts are thought to play a role related to the integration of cytoplasmic and nuclear processes influencing protein synthesis (Pardue et al., 1990; Lakhotia, 2003). hsr-Omega is likely to be an important gene as its unusual structure and several small segments of its sequence are conserved across diverse Drosophila species. In addition, chromosome puffing at its cytological location following removal from cold rearing suggests that it may also play a part in recovery from cold exposure (Lakhotia and Singh, 1985). Finally, a unique and intriguing feature of the nuclear-located omega- $n$ is a conspicuous polymorphism of a cluster of tandem repeats encoded at the $3^{\prime}$-end of the gene (Figure 1 ).
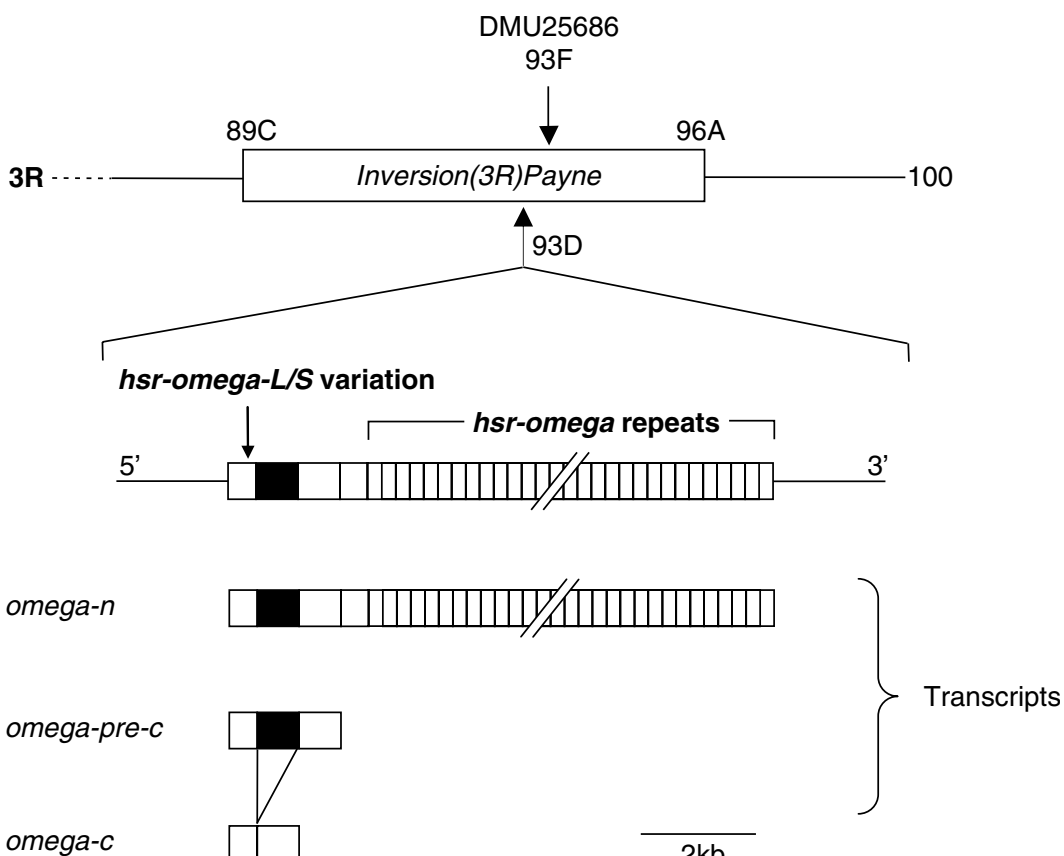

omega-c

Figure 1 Right arm of chromosome 3 (3R) showing cytological location of the hsr-omega gene (93D), In(3R)Payne and microsatellite marker DMU25686, together with a map of hsr-omega depicting the relative positions of the $5^{\prime} \mathrm{L} / \mathrm{S}$ polymorphic sites, the intron (black box) and the $3^{\prime}$ repeat variation site. The three known $h s r$-omega transcripts, including the nuclear located omega-n and cytoplasmic located omega-c, are also shown. 
The number of 280-bp repeat units, that constitute most of the omega- $n$ transcript length, varies markedly among laboratory strains, producing alleles with repeat clusters between 5 and $16 \mathrm{~kb}$ in length (Walldorf et al., 1984; Hogan et al., 1995).

In this report we investigate aspects of naturally occurring variation in the D. melanogaster hsr-omega gene. Specifically, we report on latitudinal and altitudinal hsromega repeat-number variation along a climatic gradient on the eastern coast of Australia. We assess repeatnumber association with the common cosmopolitan inversion $\operatorname{In}(3 R) P$ and with the $h s r$-omega- $L / S$ variation, in a population located at a central geographic location along the latitudinal gradient. We also look for associations of repeat number with thermal resistance traits in both a large set of family lines and a set of cold selected lines. As our data suggest an association between hsromega repeat number and both latitude and cold tolerance, we use a set of laboratory populations to examine changes in hsr-omega expression patterns following cold exposure. Our results support the hypothesis that variation in repeat number of hsr-omega is an important determining component of latitudinal variation in cold tolerance.

\section{Methods}

\section{Field samples}

Isofemale lines of $D$. melanogaster were established from field-caught females collected from banana bait trapping. Low-altitude isofemale lines were collected at regular intervals along the eastern coast of Australia spanning more than $26^{\circ}(\sim 3000 \mathrm{~km})$ in February-March of 2000, 2001 and 2002. Five high-altitude collections were made in 2002, each at a similar latitude to a different lowaltitude site. The location of collection sites and the number of isofemale lines sampled per site are indicated in Table 1 and Figure 2.

\section{Quantifying omega repeat variation}

Tandem repeat variation was genotyped by Southern blot. DNA extractions were carried out on batches of 25 flies (mixed sexes) by mortar-pestle grinding in $500 \mu \mathrm{l}$ grinding buffer $(0.2 \mathrm{M}$ sucrose, $0.1 \mathrm{M}$ Tris $\mathrm{pH} 9.2,50 \mathrm{mM}$ EDTA, $0.5 \%$ SDS), followed by the addition of $2 \mu \mathrm{l}$ of proteinase $\mathrm{K}\left(20 \mathrm{mg} \mathrm{ml}^{-1}\right)$ and incubation for $10 \mathrm{~min}$ at $45^{\circ} \mathrm{C}$. After addition of $150 \mu \mathrm{l} 8 \mathrm{M}$ potassium acetate the extract was vortexed and placed on ice for $15 \mathrm{~min}$, removed and $500 \mu \mathrm{l}$ phenol/chloroform/isoamyl alcohol (25:24:1) added and re-vortexed for $30 \mathrm{~s}$ prior to $5 \mathrm{~min}$ microfuge centrifugation at 12000 r.p.m. The supernatant was transferred to $1 \mathrm{ml}$ of $100 \%$ ethanol, vortexed and put for $4 \mathrm{~h}$ at room temperature to precipitate the DNA. Following $10 \mathrm{~min}$ centrifugation at 12000 r.p.m. the supernatant was discarded, the pellet gently washed with $70 \%$ ethanol and air dried prior to dissolving in MilliQ water overnight at $4{ }^{\circ} \mathrm{C}$. In the 2000 and 2001 collection, extractions were performed on each isofemale line. In the 2002 collection, one extraction was performed per latitudinal collection site, where each extraction consisted of five isofemale lines pooled in equal proportions (five flies per isofemale line, sexes mixed). Prior to electrophoresis DNA $\left(3.5 \mu \mathrm{g} \mu \mathrm{l}^{-1}\right)$ was double digested overnight at $37^{\circ} \mathrm{C}$ with Pst I and Hind III
Table 1 Collection sites for 2000-2002, showing the number of isofemale lines sampled for repeat-length variation each year

\begin{tabular}{|c|c|c|c|c|c|}
\hline \multirow[t]{2}{*}{ Location } & \multirow{2}{*}{$\begin{array}{l}\text { Latitude } \\
\text { (S) }\end{array}$} & \multirow{2}{*}{$\begin{array}{l}\text { Altitude } \\
(m)\end{array}$} & \multicolumn{3}{|c|}{ No. isofemale lines sampled } \\
\hline & & & 2000 & 2001 & 2002 \\
\hline Cooktown & $15^{\circ} 22^{\prime}$ & $<100$ & & 1 & \\
\hline Cape Tribulations & $16^{\circ} 01^{\prime}$ & $<100$ & & 5 & 5 \\
\hline Mossman & $16^{\circ} 27^{\prime}$ & $<100$ & 8 & 2 & \\
\hline Malanda & $17^{\circ} 19^{\prime}$ & 800 & & & 69 \\
\hline Innisfail & $17^{\circ} 30^{\prime}$ & $<100$ & 11 & 3 & 74 \\
\hline Townsville & $19^{\circ} 15^{\prime}$ & $<100$ & & 14 & 5 \\
\hline Bowen & $20^{\circ} 01^{\prime}$ & $<100$ & 7 & & \\
\hline Sarina & $21^{\circ} 25^{\prime}$ & $<100$ & 8 & 5 & 5 \\
\hline Rockhampton & $23^{\circ} 22^{\prime}$ & $<100$ & 9 & 3 & 5 \\
\hline Gladstone & $23^{\circ} 50^{\prime}$ & $<100$ & & & 5 \\
\hline Miriam vale & $24^{\circ} 19^{\prime}$ & $<100$ & 6 & & 5 \\
\hline Maryborough & $25^{\circ} 31^{\prime}$ & $<100$ & 11 & & 5 \\
\hline Rainbow Beach & $25^{\circ} 54^{\prime}$ & $<100$ & 2 & & 5 \\
\hline Redland bay & $27^{\circ} 39^{\prime}$ & $<100$ & 7 & & 5 \\
\hline Springbrook & $28^{\circ} 14^{\prime}$ & 1031 & & & 17 \\
\hline Kingscliff & $28^{\circ} 17^{\prime}$ & $<100$ & & 9 & 51 \\
\hline Duranbah & $28^{\circ} 19^{\prime}$ & $<100$ & 7 & & \\
\hline Alstonville & $28^{\circ} 50^{\prime}$ & $<100$ & 3 & & 5 \\
\hline Coffs harbour & $30^{\circ} 19^{\prime}$ & $<100$ & 11 & 11 & 58 \\
\hline Armidale & $30^{\circ} 31^{\prime}$ & 1296 & & & 67 \\
\hline Port Macquarie & $31^{\circ} 25^{\prime}$ & $<100$ & & & 5 \\
\hline Belmont & $33^{\circ} 02^{\prime}$ & $<100$ & & & 5 \\
\hline Gosford & $33^{\circ} 26^{\prime}$ & $<100$ & 7 & & \\
\hline Thirlmere & $34^{\circ} 11^{\prime}$ & $<100$ & & 2 & \\
\hline Blackheath & $33^{\circ} 38^{\prime}$ & 1046 & & & 48 \\
\hline Wollongong & $34^{\circ} 25^{\prime}$ & $<100$ & & & 53 \\
\hline Adaminaby & $35^{\circ} 59^{\prime}$ & 1025 & & & 51 \\
\hline Cobargo & $36^{\circ} 22^{\prime}$ & $<100$ & & 14 & \\
\hline Bega & $36^{\circ} 39^{\prime}$ & $<100$ & 13 & & 41 \\
\hline Yan yean & $37^{\circ} 34^{\prime}$ & $<100$ & 6 & & \\
\hline Wandin & $37^{\circ} 47^{\prime}$ & $<100$ & 4 & 5 & \\
\hline Coronet bay & $38^{\circ} 26^{\prime}$ & $<100$ & & & 5 \\
\hline Pomona & $41^{\circ} 06^{\prime}$ & $<100$ & & 4 & \\
\hline Tamar valley & $41^{\circ} 11^{\prime}$ & $<100$ & 9 & 3 & 5 \\
\hline Windara & $41^{\circ} 14^{\prime}$ & $<100$ & & 6 & \\
\hline Dilston & $41^{\circ} 19^{\prime}$ & $<100$ & & & 5 \\
\hline Red Knight & $42^{\circ} 45^{\prime}$ & $<100$ & & & 5 \\
\hline Huonville & $43^{\circ} 02^{\prime}$ & $<100$ & 8 & & \\
\hline Scenic Road & $43^{\circ} 02^{\prime}$ & $<100$ & 5 & & \\
\hline Franklin & $43^{\circ} 05^{\prime}$ & $<100$ & & 9 & \\
\hline Panorama & $43^{\circ} 07^{\prime}$ & $<100$ & & 10 & \\
\hline
\end{tabular}

(Promega, Annandale, New South Wales, Australia) in buffer B according to manufacture's protocols (thus cleaving each end of the tandem repeat region; Hogan et al., 1995). Southern blot and hybridization were performed largely as described by Sambrook et al. (1989). The DNA was transferred to a Zeta-probe membrane (Bio-Rad, Gladesville, New South Wales, Australia) and probed with $\gamma-{ }^{32} \mathrm{P}$ radio-labelled $D$. melanogaster repeat units. The radioactive membrane was exposed to X-ray film and developed in an automated X-ray machine.

As there were a large number of different band positions (alleles), some of which were difficult to distinguish, they were analysed in the 2000 collection by placing alleles into five different groups based on size. A size standard, containing four different repeat-length alleles spaced apart at 20.3, 14.5, 10.6 and $8.7 \mathrm{~kb}$ (created by pooling DNA from two isofemale lines) was electrophoresed on all gels in at least two well positions (Figure 3a). The size of the four fragments in the standard 


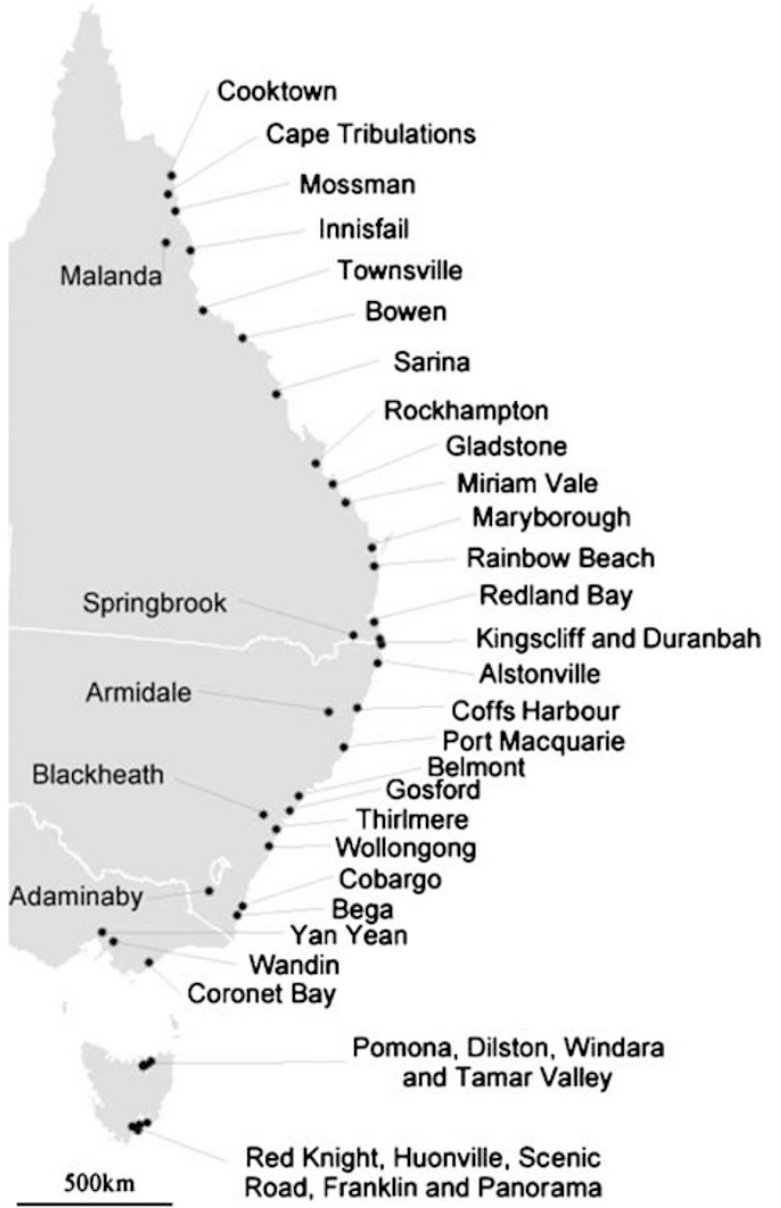

Figure 2 Collection sites for the latitude and altitude survey of $h s r$ omega repeat-length (text on right-hand side of coastline represents low altitude; left-hand text represents high altitude).

were estimated by comparing the autoradiograph with marked origin to a captured image of the original gel, adjacent to a ruler scale, on which molecular weight size markers (Expand DNA mw marker XV; Roche Diagnostics, Castle Hill, New South Wales, Australia) were labelled with ethidium-bromide. The size standard allowed the placing of alleles into five groups: A $(>20.3 \mathrm{~kb}), \mathrm{B}(>14.5$ to $\leqslant 20.3 \mathrm{~kb}), \mathrm{C}(>10.6$ to $\leqslant 14.5 \mathrm{~kb}), \mathrm{D}(>8.7$ to $\leqslant 10.6 \mathrm{~kb})$ and $\mathrm{E}(\leqslant 8.7 \mathrm{~kb})$. The mean band size for each group being estimated at $20.8,17,12.6,9.7$ and $6 \mathrm{~kb}$, respectively. These size means were used to estimate the average number of repeats for each group, corresponding to $63,49,34,23$ and 20 repeats, respectively (as each repeat is $283 \mathrm{bp}$ in length and each fragment contains $\sim 3 \mathrm{~kb}$ of flanking region; Hogan et al., 1995).

To estimate the average number of repeats for a sample the allele group frequency (the proportion of bands falling in that group) was multiplied by the average repeat number for that group and these were summed across groups. Average repeat number for a location was the mean value of all isofemale lines. In 2001 and 2002 alleles were also grouped. However, to improve resolution total group band intensity was measured (the sum of the intensities of bands within each group-group intensities summing to one for the gel lane). This was performed by scanning bands from the X-ray film using a
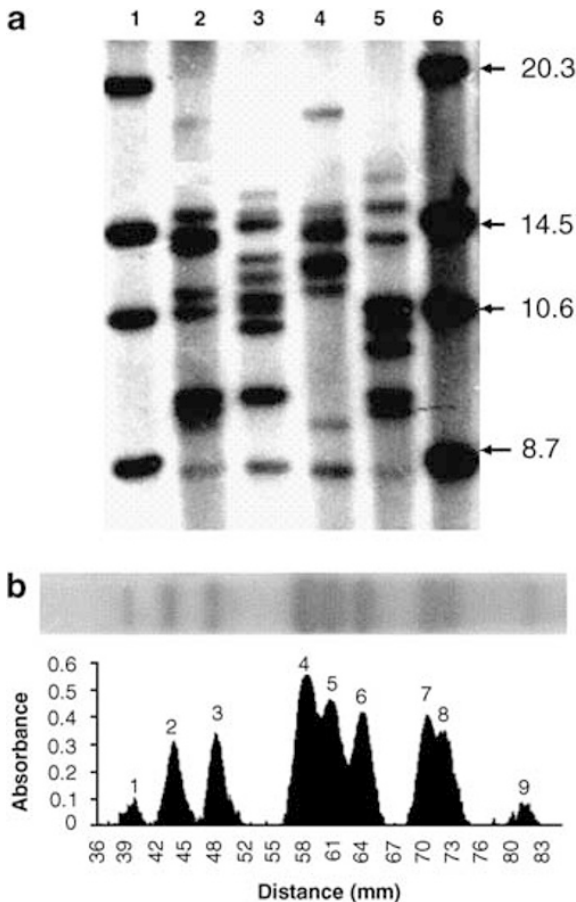

Figure 3 (a) Southern blot gel image of restriction-digested DNA probed with hsr-omega repeat sequence. Lanes 1 and 6 are standard size markers whereas lanes $2-5$ show typical repeat-length variation each from five pooled isofemale lines. (b) Absorbance scan of lane 5 from (a) used to determine band positions and densities to weight allele groups.

a Bio-Rad imaging densitometer (Model GS-700) and weighted using Molecular Analyst Version 1.4 software that provided relative differences in band intensities (Figure $3 b$ ). The frequency of the allele group for the sample was estimated by dividing group intensity by average repeat number for that group (as the probe detects individual repeats and band intensity will depend on the number of repeats), summing these and expressing each group as a proportion. Average repeat number of a sample was then estimated as for 2000. This method only provides an estimate of average repeat number and frequency of an allele group for a sample as two different-sized fragments (with different repeat number) within a group are not discerned. However, this has proved to be an accurate and reliable protocol, especially for the 2002 collection and for the linkage disequilibrium study, when agarose gels were run for longer to resolve a larger number of different alleles and 10 different groups were distinguished (average band size and group repeat number being adjusted accordingly).

Average number of repeats for a location was analysed as a general linear model fitting year, latitude and year by latitude, using SPSS for Windows (14). Altitudinal variation of average number of repeats was assessed by paired comparisons of a high- with a low-altitude location at each of five latitudes (Figure 2) using a three-way $\chi^{2}$ analysis (Cochrans-Mantel-Haensel (CMH) test; Sokal and Rohlf, 1996; SPSS 11.5). The Australian Bureau of Meteorology web site (www.bom.gov.au) was used to obtain six climatic variables (average daily maximum temperature for the hottest month, average 
daily minimum temperature for the coldest month, average annual number of days above $30^{\circ} \mathrm{C}$, average annual number of days below $2{ }^{\circ} \mathrm{C}$, mean monthly rainfall for the wettest month and mean monthly rainfall for the driest month). These were examined for association (correlation and partial correlation analyses using SPSS 14) with the average repeat-number data across all collection sites (3 years combined).

\section{Family lines trait associations}

Using a set of single-pair-mating family lines derived by Weeks et al. (2002), variation in hsr-omega repeats was examined for associations with three traits, cold tolerance (chill-coma recovery time), heat resistance (knockdown time) and wing size, traits that were characterized for these families three generations earlier (Weeks et al., 2002). Southern blots were carried out on DNA extracted from 25 adults from each family line which was scored for both the frequency of $f r$ (few-repeat) alleles (= combined frequency of band groups containing bands $<10.6 \mathrm{~kb}$ ) and the frequency of $m r$ (many-repeat) alleles (= combined frequency of groups containing bands $>14.5 \mathrm{~kb}$ ), determined by comparison to bands of standard sizes run on every gel. Data were analysed using both Pearson's coefficient and Spearman's coefficient of rank correlation in SPSS (11.5).

\section{Cold-tolerant selection lines}

A set of three cold-tolerant populations and three unselected controls, previously derived by Anderson et al. (2005b), were assayed for hsr-omega repeat variation, and for tissue levels of hsr-omega transcripts. Briefly, these independent populations were selected every second generation for fast chill-coma recovery time starting with a mass-bred population formed by pooling 25 isofemale lines derived equally from five low latitude sites on the north-eastern Australian coast (from latitudes between $16^{\circ} 01^{\prime}$ and $23^{\circ} 50^{\prime}$ ). DNA was extracted for the six populations at generation 22, when chill-coma recovery time averaged $\sim 65 \%$ that of the control populations (Anderson et al., 2005b) and hsr-omega repeats quantified as described above. Levels of omega$n$ and omega-c were determined by real-time reverse transcriptase (RT)-PCR. A total of 3 replicate RNA extracts were made from all lines, each using 30 adult female flies and TriSure reagent (Bioline, Alexandria, New South Wales, Australia). Reverse transcription was completed with $2 \mu \mathrm{g}$ of total RNA primed with $200 \mathrm{ng}$ random primers (Invitrogen, Mt Waverley, Victoria, Australia) and using BioScript reverse transcriptase (Bioline). Real-time PCR was performed in the RotorGene 6000 (Corbett Lifescience, Mortlake, New South Wales, Australia) for omega- $n$ and omega-c. Measures were internally normalized to levels of cyclin $K$ chosen because in microarray studies it showed particularly low variance across stress treatments, especially heat treatment, and it occurred at appropriate levels for hsr-omega transcript estimates (Sørensen et al., 2005). Duplicate PCR reactions using transcript-specific primers $(400 \mathrm{nM})$ for omega-n (F-5'-TCCGCATTTATTTTTCTCCAC-3', R-5'-GTGTATAGAATTTGGGACCTCCA-3'), omega-c (F-5'-TAGGAAGCCAGTGGGCGT-3', R-5'-CCGAGTG CGTTTTCAGCA-3') and for cyck (F-5'-GAGCATCCT TACACCTTTCTCCT-3', R-5'-TAATCTCCGGCTCCCA
CTG-3') were completed for each cDNA batch using the Sensimix DNA kit (Quantace, Alexandria, New South Wales, Australia). Amplification conditions were $95^{\circ} \mathrm{C} 10 \mathrm{~min}$ followed by 50 cycles of $95^{\circ} \mathrm{C}(10 \mathrm{~s}), 58^{\circ} \mathrm{C}$ (15s) and $70{ }^{\circ} \mathrm{C}(5 \mathrm{~s})$ with fluorescence data acquisition at the completion of the $70^{\circ} \mathrm{C}$ step every cycle. Following cycling, product melt curves were generated by raising temperature from 69 to $90^{\circ} \mathrm{C}$ in $1{ }^{\circ} \mathrm{C}$ increments. Primer pairs were previously validated across a range of cDNA concentrations with efficiencies found to be within the acceptable ranges. For transcript level analysis, $\mathrm{Ct}$ differences between cyck and omega- $n$ and cyck and omega-c for each RNA extract were converted to fold differences and nested analysis of variance (ANOVA; SPSS 14.0) used to determine the effects of line and selection treatment.

\section{Linkage disequilibrium}

Adult D. melanogaster were collected from Coffs Harbour $\left(30^{\circ} 19^{\prime} \mathrm{S}, 153^{\circ} 08^{\prime} \mathrm{E}\right)$ in March 2001 using banana bait traps and 50 isofemale lines (50) were set up at $18^{\circ} \mathrm{C}$ using the field caught females. In order to score the haplotype of individual third chromosomes from this sample, a crossing scheme was established that allowed 78 independently derived chromosomes to be isolated. $h s r-O m e g a-L / S$ scoring during the derivation of these lines allowed two independent chromosomes to be obtained from isofemale lines where the female was heterozygous. For each of the 50 lines several F1 males were single-pair mated to a virgin female carrying a chromosome three-balancer chromosome (TM6B). The parent F1 males were genotyped for hsr-omega-L/S to determine which lines to use for a second cross that would maximize the number of independent chromosomes assessed. Several male progeny that carried TM6B (with tubby and humeral phenotype) were individually crossed to virgin females from a stock that was homozygous for the markers of interest. This cross isolated independent chromosomes in separate fly lines. Within each line progeny that did not contain the balancer phenotype were used for scoring three markers on the right arm of chromosome three; hsr-omega-L/S, the closely linked microsatellite DMU25686, and $\operatorname{In}(3 R) P$. hsr-Omega-L/S and DMU25686 were genotyped using previously described methods (Gockel et al., 2001; Weeks et al., 2002). In(3R)P was scored by polytene chromosome squashes and confirmed by a PCR-product gel assay (Anderson et al., 2005b).

Linkage disequilibrium between pairs of clinal markers was assessed using the standardized linkage disequilibrium coefficient $D^{\prime}$ (Hedrick, 2000); $D^{\prime}$ was calculated using the program Haploxt (Abecasis and Cookson, 2000) and the significance of linkage disequilibrium was determined using Fisher's exact test (Raymond and Rousset, 1995). P-values were corrected for multiple comparisons using the Sequential Bonferroni method (Rice, 1989). Linkage disequilibrium $\left(D^{\prime}\right)$ was also estimated between $h s r$-omega-L/S and hsr-omegarepeats in the family lines and significance assessed using Spearman's coefficient of rank correlation in SPSS.

\section{hsr-Omega expression and cold shock}

Three mass-reared laboratory populations were established 2 years prior to sampling, and kept in the laboratory at $25^{\circ} \mathrm{C}$ in triplicate bottle culture for each 
population. Each population was derived from an independent sample of $\sim 50$ adults from a homogenous pooling of 25 isofemale lines collected from several lowlatitude $\left(<24^{\circ} \mathrm{S}\right)$ and low-altitude $(<100 \mathrm{~m})$ coastal sites from Queensland, Australia. Three groups of 2 to 6-dayold females $(\sim 30)$ from each of the three populations were exposed to $0{ }^{\circ} \mathrm{C}$ for $6 \mathrm{~h}$ and allowed to recover for $20 \mathrm{~min}$ at $25^{\circ} \mathrm{C}$ before being frozen for RNA extraction. For controls, three groups of un-exposed females from each population were collected in parallel. Real-time RTPCR was used to determine levels of both transcripts, as described. To determine affects of population and cold treatment, nested ANOVA was performed for both omega- $n$ and omega-c, separately (SPSS 14.0).

\section{Results}

\section{Geographical variation}

Within Australian D. melanogaster a large number of allele sizes were detected (Figure 3). It was not unusual to detect more than 10 different sized fragments, from a single population sample. The fragments from natural populations along the eastern coast of Australia varied from $\sim 8 \mathrm{~kb}$ to at least $20 \mathrm{~kb}$ ( $\sim 18$ to $\sim 61$ repeat units, given that the fragments contained $\sim 3 \mathrm{~kb}$ of flanking sequence) with an average of $\sim 12 \mathrm{~kb}$ ( $\sim 32$ repeats).

The average number of repeats showed a clear linear effect of latitude $\left(\mathrm{F}_{1,50}=32.6, P<0.001\right)$ that was consistent across years (no year by latitude interaction, $\mathrm{F}_{2,50}=0.09, P=0.91$ ), and did not depend on the year sampled (there was no effect of year, $F_{2,50}=0.52$, $P=0.60)$. Quadratic terms did not improve the fit of the data. The association with latitude was negative; alleles with a lower number of repeats occurred more frequently at higher latitudes (Figure 4). We did not detect an effect of altitude on average number of repeats $\left(\chi_{\mathrm{CMH}}^{2}=0.77,1\right.$ degrees of freedom, d.f., $\left.P>0.05\right)$, and no interaction with latitude occurred in a three-way $\chi^{2}$ analysis. Using meteorological data assembled from all low-latitude sites (within $20 \mathrm{~km}$ of the collection sites) we looked for associations of average repeat number with four average temperature and two rainfall statistics. Although significant Pearson correlations occurred for all climatic variables, except mean monthly rainfall for the

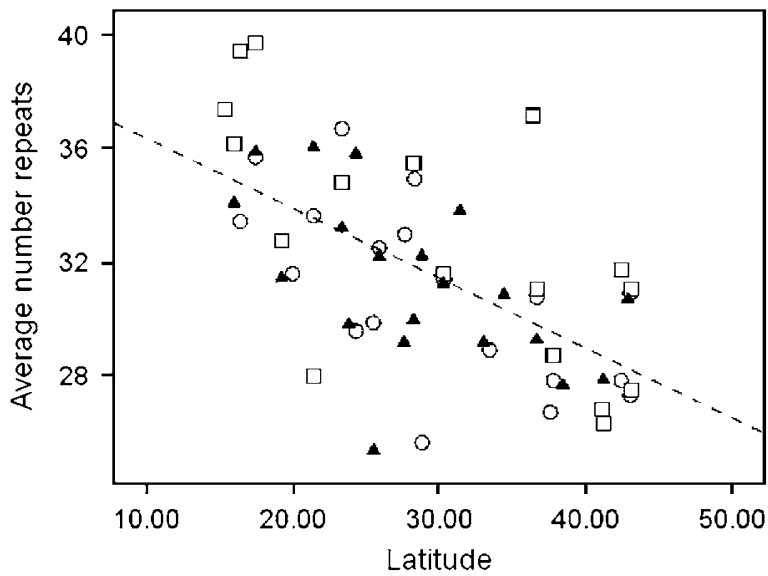

Figure 4 Association of average number of repeats with latitude in $2000\left(\bigcirc ; R^{2}=0.42, t=-3.48, P<0.01\right), 2001\left(\boldsymbol{\Lambda} ; R^{2}=0.48, t=-3.72\right.$, $P<0.01)$ and $2002\left(\square ; R^{2}=0.32, t=-2.91, P=0.01\right)$. driest month, in partial correlation analyses these all became low and non-significant when latitude was controlled. Adding the high-altitude sites to these analyses did not significantly increase the levels of association detected.

\section{Family lines trait association}

To look for any association of the repeat variation with thermal tolerance traits we characterized a set of family lines for hsr-omega repeat-number variation using Southern blots and measured chill-coma recovery time and heat resistance (Table 2). Families with a higher frequency of few-repeat ( $f r)$ alleles were more cold tolerant-their chill-coma recovery time was shorter (Figure 5), but there was no association of $f r$ allele frequency with heat resistance. For the many-repeat $(m r)$ allele group there was no indication of an association with either chill-coma recovery time or heat resistance.

We also examined hsr-omega repeat-number variation for association with wing area in the family line study as the earlier report on these lines (Weeks et al., 2002) found hsr-omega-S variation to be negatively associated with wing area (Table 2), and a major genetic factor that contributes to wing size variation maps to the vicinity of

Table 2 hsr-Omega genotype associations (Pearson's $r$ ) with three clinal traits for Coffs Harbour family lines

\begin{tabular}{lcc}
\hline Trait & $\begin{array}{c}\text { Average number of } \\
\text { repeats; hsr-omega-fr } \\
\text { allele group frequency }\end{array}$ & $\begin{array}{c}\text { Omsr- } \\
\text { Omega-S }\end{array}$ \\
\hline Heat knockdown & $0.020(101) ;-0.057^{\mathrm{b}}$ & $-0.085(104)$ \\
resistance time & & \\
Chill-coma recovery time & $0.178^{*}(102) ;-0.224^{\mathrm{b}, *}$ & $-0.037(104)$ \\
Wing area & $0.193^{*}(94) ; 0.234^{\mathrm{b}, *}$ & $-0.472^{* * *}(96)$ \\
\hline
\end{tabular}

Numbers in parenthesis indicate number of family lines compared. ${ }^{\text {a }}$ Data from Weeks et al. (2002).

'Spearman's rank correlation coefficients.

${ }^{*} P<0.05,{ }^{* * *} P<0.001$

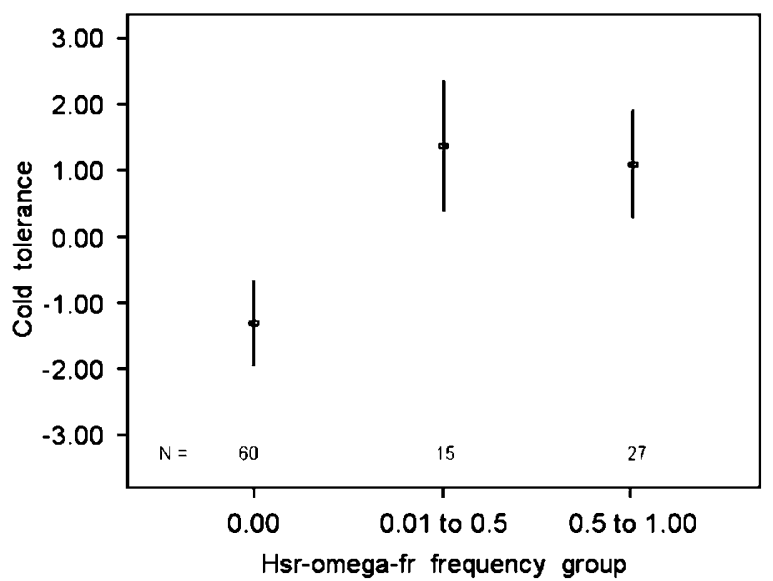

Figure 5 Associations in the Coffs Harbour family lines between $h s r$-omega repeat-number variation (lines placed into three $h s r$ omega-fr allele frequency groups) and cold tolerance (chill-coma recovery time (standardized) $x-1 \pm$ s.e.). The number of families per group are indicated $(N)$. Analysis of variance (ANOVA) indicates a significant effect of hsr-omega-fr frequency group $\left(\mathrm{F}_{2,99}=3.32\right.$, $P<0.05)$-family lines with higher frequencies of few-repeat alleles having high cold tolerance. 
Table 3 Number of hsr-omega tandem-repeat bands, the average number of repeats and frequency of markers $h s r-o m e g a-L, I n(3 R) P$ and DMU25686 present in control (C1-C3) and cold-selected (S1-S3) lines

\begin{tabular}{|c|c|c|c|c|c|}
\hline Line & $\begin{array}{l}\text { Average number } \\
\text { repeats (s.e.) }\end{array}$ & $\begin{array}{l}\text { Number of gel bands } \\
\text { (= number of alleles) }\end{array}$ & $\begin{array}{l}\text { Frequency } \\
\text { hsr-omega-L }\end{array}$ & $\begin{array}{l}\text { Frequency }^{\mathrm{a}} \\
\operatorname{In}(3 R) P\end{array}$ & $\begin{array}{l}\text { Frequency }^{\mathrm{a}} \text { DMU } 25686 \\
\text { (most common allele) }\end{array}$ \\
\hline $\mathrm{C} 1$ & $40.44(4.82)$ & 5 & 0.84 & 0.15 & 0.15 \\
\hline $\mathrm{C} 2$ & 41.44 (11.39) & 7 & 0.89 & 0.15 & 0.36 \\
\hline C3 & $44.07(5.05)$ & 6 & 0.65 & 0.33 & 0.34 \\
\hline S1 & $38.83(3.16)$ & 4 & 0.5 & 0.48 & 0.53 \\
\hline S2 & $38.73(3.58)$ & 4 & 0.79 & 0.24 & 0.5 \\
\hline S3 & $39.31(4.85)$ & 4 & 0.7 & 0.22 & 0.28 \\
\hline
\end{tabular}

${ }^{a} n=30$ flies/marker/line.

hsr-omega (Calboli et al., 2003). Larger body size (often approximated as wing size) is recognized as an adaptation to cold-temperature environments and clinal variation in body size (Avis et al., 1997) has been associated with clinal variation in $\operatorname{In}(3 R) P$ (Rako et al., 2006) that is in linkage disequilibrium with hsr-omega-L/S (Anderson et al., 2005a). A positive hsr-omega-fr allele frequency association with wing area was evident (Table 2). However, this was not robust as a partial correlation between $f r$ allele frequency and wing size after controlling for $h s r$-omega-S was not significant $(r=0.104$, d.f. $=88, P=0.16)$. The partial correlation between $h s r$ omega-S and wing area, controlling for hsr-omega-fr allele frequency, remained highly significant, $r=-0.42$, $P<0.001$ (d.f. =88). For the hsr-omega-mr allele group there was no indication of an association with wing area. The data therefore suggest that hsr-omega repeat variation is independent of wing area.

\section{Selected cold-tolerant populations and hsr-omega variation}

A set of six populations, three selected for fast chill-coma recovery time and three non-selected control populations, were examined for hsr-omega repeat-number variation. Lines selected for cold tolerance showed a significantly lower average number of repeats when compared to unselected control lines (Table 3; $F_{1,4}=8.96$, $P=0.04$ ). There was also a significant line effect in these samples $\left(\mathrm{F}_{4,30}=3.81, P=0.013\right)$. No association of linked variation in $h s r$-omega-L/S, $\operatorname{In}(3 R) P$ or the microsatellite marker DM25686 occurred with the cold-selected or control populations (Table 3).

A significant difference in constitutive levels of the omega- $n$ transcript between cold-selected and control lines (Figure 6a) was revealed by nested ANOVA $\left(\mathrm{F}_{1,4}=6.00, P=0.031\right)$, with no effect of line within selection treatments $\left(\mathrm{F}_{1,12}=0.76, P=0.57\right)$. On average, cold selected lines displayed a $14 \%$ reduction in expression compared to control lines. No differences in expression between lines or selection treatments $\left(\mathrm{F}_{1,4}=0.554, P=0.498\right)$ was detected for omega-c. Thus, two changes that reduce the number of hsr-omega- $n$ transcript-bearing repeat units in tissues have occurred in the cold-tolerant populations due selection for cold tolerance-alleles with fewer repeat units have increased in frequency and the tissue level of this transcript is lower.

\section{Linkage disequilibrium}

In an attempt to tease apart associations between $h s r-$ omega repeat variation, thermal traits and closely linked
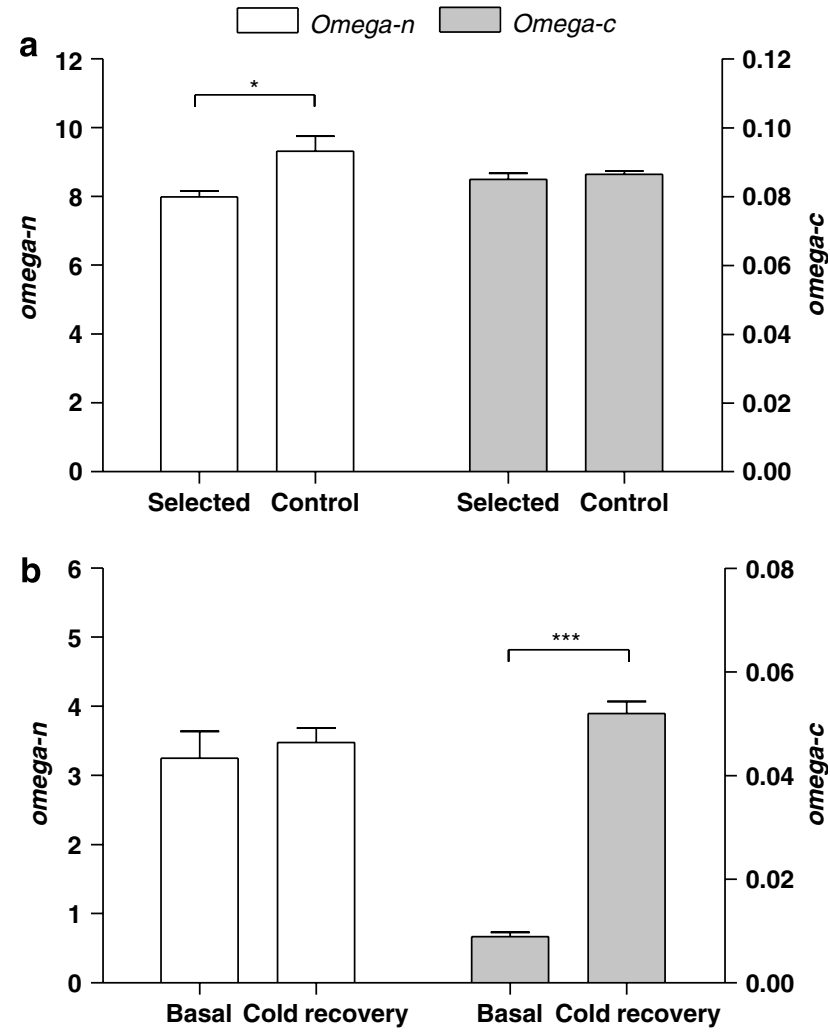

Figure 6 Cold treatment-related changes in levels of omega- $n$ and omega-c transcripts determined by quantitative reverse transcriptase (qRT)-PCR. Bars represent the mean transcript levels normalized to cyck \pm 1 standard error. (a) Comparison of the constitutive levels of both transcripts in adult tissues in cold-resistant (selected) populations vs cold-sensitive (control) populations. Only hsr-omega- $n$ levels differed between selected and control populations $\left(\mathrm{F}_{1,4}=6.00\right.$, ${ }^{*} P=0.031$ ). (b) Comparison of basal levels (adults held at $25^{\circ} \mathrm{C}$, no cold treatment) to cold recovery levels of both transcripts in adult tissues following exposure to $0{ }^{\circ} \mathrm{C}$ for $6 \mathrm{~h}$, then recovery for $20 \mathrm{~min}$ at $25^{\circ} \mathrm{C}$ for the set of three mass-reared laboratory populations $\left(\mathrm{F}_{1,4}=539.3,{ }^{* * *} P<0.001\right)$.

markers that also associate with these traits, linkage disequilibrium $\left(D^{\prime}\right)$ was evaluated in a population recently collected from a location that is central along the latitudinal transect, Coffs Harbour. A central population was chosen because the markers also show latitudinal frequency clines and the rarity of genotypes present at high and low latitudes render significant disequilibrium difficult to detect. Fisher's exact test was used to determine the significance of pairwise comparisons between four markers that are closely 

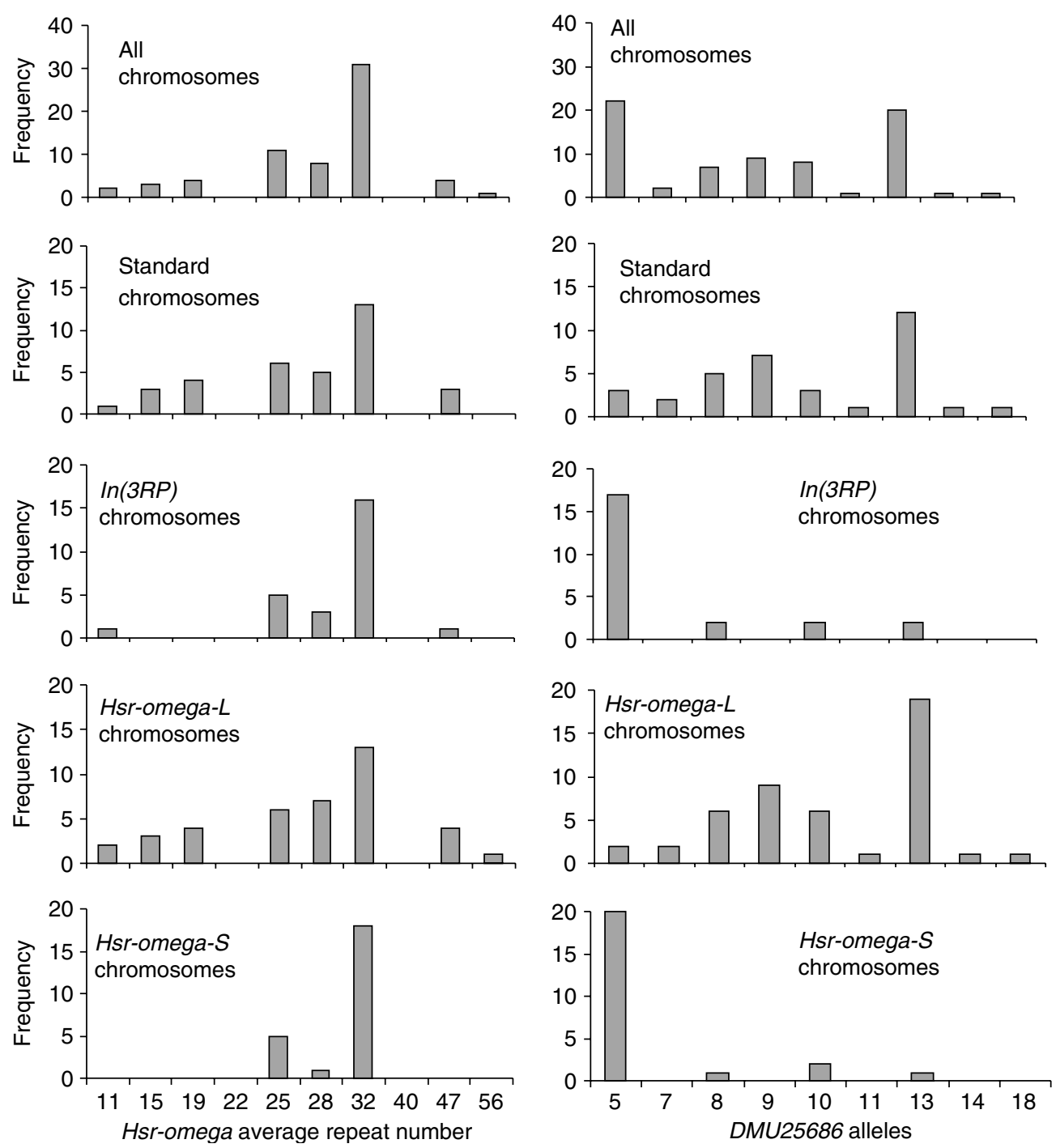

Figure 7 Gamete frequency distributions of hsr-omega repeat allele groups (on left-hand side, LHS), and DMU25686 alleles (on right-hand side, RHS), on selected third chromosomes from the Coffs Harbour population.

linked on the right arm of chromosome 3, hsr-omega repeats, $\operatorname{In}(3 R) P$, hsr-omega-L/S and the microsatellite $D M U 25686$. A significant linkage disequilibrium was observed between hsr-omega-L/S and $\operatorname{In}(3 R) P\left(D^{\prime}=0.72\right.$, $P<0.001$ ), as reported previously (Anderson et al., 2005a). Of 36 standard chromosomes sampled only 4 (11\%) carried the hsr-omega-S allele, whereas 21 of 27 (78\%) inversion chromosomes carried the $S$ allele. Also, as depicted in Figure 7, strong linkage disequilibrium occurred between hsr-omega-L/S and DMU25686 $\left(D^{\prime}=0.77, \quad P<0.001\right)$, and between DMU25686 and $\operatorname{In}(3 R) P \quad\left(D^{\prime}=0.62, P<0.01\right)$. However, although $h s r-$ omega repeat-number variation was not significantly associated with the inversion, or with DMU25686, it was mildly associated with hsr-omega-L/S $\left(D^{\prime}=0.57\right.$, $P=0.05)$. There was a positive association between $h s r-$ omega- $L$ and allele groups that had both very low- and very high-repeat number (Figure 7). In this sample the hsr-omega-S chromosomes did not have repeat numbers in the high or low part of the range.
hsr-Omega expression and cold shock

To assess whether or not the transcripts of this gene were regulated in response to cold stress we quantified their levels in tissues $20 \mathrm{~min}$ into recovery from a 6-h exposure of adults to $0{ }^{\circ} \mathrm{C}$, and compared them to that from adults held at $25^{\circ} \mathrm{C}$. Although no obvious change was detected in omega- $n$ levels, a large increase occurred in the level of omega-c $\left(\mathrm{F}_{1,4}=539.28, P<0.001\right)$, about a sixfold increase (Figure 6b). Although our method of transcript quantification was not absolute the data suggest that the levels of omega- $n$ were higher than that of omega-c (Figure 6), in contrast to a previous report where the levels detected were similar (McKechnie et al., 1998).

\section{Discussion}

To our knowledge repeat-number variation of the Drosophila hsr-omega gene from natural populations has not been previously investigated. Hogan et al. (1995) reported that the length of the repeat fragment that we 
used in this investigation varied among laboratory stocks of $D$. melanogaster, from between 5 and $16 \mathrm{~kb}$. Our data reveal that natural populations of this species, in Australia at least, contain large numbers of alleles with marked variation in repeat number, and with high levels of heterozygosity. Similar to the laboratory stock data of Hogan et al. (1995) the repeat fragment in Australian samples showed a lower length limit of $\sim 5 \mathrm{~kb}$, however, we observed a higher upper limit of about $20 \mathrm{~kb}$ of repeats. This is likely to be close to the upper limit for the species as over 300 isofemale lines were scored from a wide range of latitudes.

Our sampling has established that repeat-number variation shows a robust latitudinal cline along the eastern coast of Australia, with the average number of repeats being negatively associated with latitude. Temporal analysis of this cline shows that it is consistent over 3 years suggesting that average repeat number is relatively stable in Australian populations. As several other closely linked and highly variable genetic markers do not show latitudinal clines along this same Australian transect (Gockel et al., 2001) the stable latitudinal repeat cline suggests that natural selection is acting on this region of the chromosome. Note however that we did not detect any association between average repeat number and altitude, which one might have expected if environmental temperature was a factor that influenced the population frequency of different repeat-number alleles. However with altitude, as distinct from latitude, gene flow might account for a lack of differentiation over the shorter distances associated with habitat temperature variation. Also, we failed to detect any robust association of the hsr-omega repeat variation with any of the climatic variables assessed over the sampled distribution.

A cline itself does not exclude the possibility that hsr-omega repeat variation is adaptively neutral and a product of hitch-hiking with closely linked adaptive variation in another gene. The strongest candidates for such an adaptive marker, in linkage disequilibrium with the repeat variation, are the breakpoints of the cosmopolitan inversion $\operatorname{In}(3 R) P$ (Kennington et al., 2006). However, we consider this is an unlikely possibility as we have shown that the repeat variation is largely independent of $\operatorname{In}(3 R) P$, despite being contained within it (Figure 7). Also, the repeat-number variation was not in linkage disequilibrium with a closely linked and clinally varying microsatellite marker DMU25686, that itself was in strong linkage disequilibrium with both $h s r$-omega-L/S and $\operatorname{In}(3 R) P$, consistent with a previous report (Weeks et al., 2002). Mild linkage disequilibrium was detected between the repeats and hsr-omega-L/S, however as depicted in Figure 7, this association seems 'nondirectional' in that alleles that that have both few repeats and many repeats were both positively associated with the $h s r$-omega- $L$ allele that occurs at high frequency only at higher latitudes (the hsr-omega-S chromosomes had an absence of both few and many repeats). Thus, if the hsromega- $L$ allele is held at high frequency at cooler latitudes by selection this would not obviously result in high frequency of alleles with few repeats, assuming the same central-population pattern of linkage disequilibrium among these markers occurs at higher latitudes. Hence, our data are consistent with the idea that the hsr-omega repeat marker variation is largely independent of variation in the closely linked markers DMU25686 and
In(3R)P, and that a level of repeat disequilibrium with the clinally varying hsr-omega-L/S variation cannot 'explain' the cline in the repeats.

Although several independent data sets have shown an association of hsr-omega-L/S variation with heat knockdown tolerance (McColl et al., 1996; McKechnie et al., 1998; Anderson et al., 2003; Rako et al., 2007), and one QTL study (Norry et al., 2004) was consistent with an hsr-omega effect, some studies have not found hsr-omega variation to be associated with heat tolerance or heat stress. This may be because different measures of heat tolerance have been used (for example Morgan and Mackay, 2006) or because relevant microarray studies have used arrays on which hsr-omega is not represented (Leemans et al., 2000; Sørensen et al., 2005). Our central population family study gave no indication of an association of hsr-omega repeat variation with knockdown heat resistance. Although this might be because we only measured basal heat knockdown tolerance, not heat-hardened or mortality-based heat tolerance, these data did suggest an association between cold-tolerance and hsr-omega repeat variation-family lines that contained more few-repeat alleles had shorter chill-coma recovery times. This association is in the expected 'adaptive' direction as cold tolerance increases with latitude (Hoffmann et al., 2002), as does the frequency of alleles with low repeat number. Limited repeat variation data from a previous report is consistent with fewer repeats being associated with increased cold tolerance (measured both by reduced cold mortality and faster chill-coma recovery time; Anderson et al., 2003). However, we did not detect a strong association and further investigation was called for.

We therefore examined repeat variation in a set of three replicate lines selected for fast recovery from chillcoma for 22 generations, and in three non-selected control lines, and found an association in the expected direction, the cold-resistant lines had, on average, alleles with fewer repeats than the control cold-sensitive lines. Genotype variation between these cold-resistant lines and their controls indicated no association with the closely linked clinal markers, In(3R)P, DMU25686 or hsromega- $L / S$. If the repeat variation and cold tolerance are causally related these findings for closely coupled markers are not surprising as linkage disequilibrium patterns are consistent with this interpretation. Also, the 'non-directional' linkage disequilibrium of the repeat and hsr-omega-L/S variations might explain why, when repeat association was detected between both coldtolerance and hsr-omega-L/S variation, no association was detected between cold-tolerance and hsr-omega-L/S variation, both in these cold-selected populations and in the family line data.

The hsr-omega gene is clearly one of the Drosophila heatstress genes as, like the heat-shock protein genes, it is upregulated in response to mild heat shock (Pardue et al., 1990). We asked if its regulation is also affected by cold treatment. Unlike other heat-shock puffs of D. melanogaster, the hsr-omega locus (at cytological location 93D) responds by puffing when a brief cold shock is given to warm-reared larvae (Singh and Lakhotia, 1984), and when cold-reared larval salivary glands are given a brief $24{ }^{\circ} \mathrm{C}$ warm-shock puffing occurs at the 93D locus and not at the puff sites producing heat-shock proteins (Lakhotia and Singh, 1985). However, puffing itself does 
not definitively implicate the hsr-omega gene as several genes locate to the puff sites.

In this study we have shown upregulation of the omega-c transcript of hsr-omega following a 6-h exposure to $0{ }^{\circ} \mathrm{C}$ which suggests that the gene itself is involved in an adaptive response to cold. Although we only examined one time point, $20 \mathrm{~min}$ into recovery from the cold exposure, our data indicated a large increase in omega-c levels, and that this did not occur for the omega- $n$ transcript. A temporal expression study during cold recovery in this species, as was recently performed by microarrays on which hsr-omega was not represented (Qin et al., 2005), is needed for both hsr-omega transcripts. We also found that constitutive levels of omega- $n$ were reduced in replicate populations selected for cold tolerance, suggesting a role for omega- $n$ in determining fitness following cold exposure. Both transcripts of this gene may help implement altered cellular processes that facilitate cold acclimation or efficient cold recovery, or both. Although our expression data implies a role for $h s r-$ omega in a response to cold exposure, we cannot exclude the possibility that an unidentified closely linked gene in linkage disequilibrium with hsr-omega repeat variation has caused the association between repeat-number variation and cold tolerance.

Nonetheless to our knowledge this is the first study to demonstrate an association between genetic variation in hsr-omega repeats and both geographic and thermal environmental factors. Our data suggest that the repeat variation affects fitness in cold environments, that it is selected upon by some aspect of naturally occurring cold exposure, and that the consequence is a latitudinal genetic cline in frequency of alleles that encode different numbers of repeats-fewer repeats being favoured at colder latitudes. Just what cellular or physiological mechanisms might be responsible for such an association or process awaits further research. We can speculate however, as considerable background knowledge at the molecular and cellular level is available. The hsr-omega gene produces two major RNA transcripts, in all tissues, but it does not produce a detectable protein product. Although overall sequence divergence is not high across the Drosophila genus, gene structure and several short regions of sequence are highly conserved (Pardue et al., 1990). One such highly conserved sequence, across at least 12 Drosophila species, is a nonamer motif (AUAGGUAGG), two of which are imbedded in each $280 \mathrm{bp}$ monomer of the repeats (Bardsley L and McKechnie SW, unpublished). Evidence suggests that these motifs, that are part of the nuclear transcript, may associate with heterogeneous ribonuclear proteins (hnRNPs) and help coordinate the availability of hnRNPs for messenger RNA processing ( $\mathrm{Zu}$ et al., 1998). In relation to the latitudinal cline, populations at cooler latitudes on average have fewer repeating nonamers (shorter omega$n$ transcripts) and therefore would have few binding sites to sequester hnRNPs with possible downstream affects on rates of messenger RNA processing and protein synthesis. Such effects might be expected to increase when thermal stress impinges on the individual and the gene is upregulated. However, further work is needed to investigate cellular processes affected and concomitant fitness effects.

Regardless of any mechanism the data presented here are consistent with a model where-by hsr-omega repeat number is a causal factor underlying the established Australian latitudinal cline in chill-coma recovery time in this species. These data also suggest that hsr-omega, although recognised for some time as being involved in the cellular heat-stress response and being associated with heat-tolerance variation, may also be important in the cell's response to cold stress and influence the organism's performance in colder environments.

\section{Acknowledgements}

We thank Michelle Schiffer for her field collection efforts, Ary Hoffmann for valuable discussion and help with analyses, and Nicole DeRycke and John Arvanitakis for help with experimental work. We are also grateful to three reviewers for their constructive suggestions that significantly improved the paper. The project was supported by the Australian Research Council by their Special Research Centre Program.

\section{References}

Abecasis GR, Cookson WO (2000). GOLD—graphical overview of linkage disequilibrium. Bioinformatics 16: 182-183.

Anderson AR, Collinge JE, Hoffmann AA, Kellett M, McKechnie SW (2003). Thermal tolerance trade-offs associated with the right arm of chromosome 3 and marked by the hsr-omega gene in Drosophila melanogaster. Heredity 90: 195-202.

Anderson AR, Hoffmann AA, McKechnie SW, Umina PA, Weeks AR (2005a). The latitudinal cline in the In(3R)Payne inversion polymorphism has shifted in the last 20 years in Australian Drosophila melanogaster populations. Mol Ecol 14: 851-858.

Anderson AR, Hoffmann AA, McKechnie SW (2005b). Response to selection for rapid chill-coma recovery in Drosophila melanogaster: physiology and life-history traits. Genet Res Camb 85: 15-22.

Avis CJ, Azevedo RBR, Partridge L (1997). Genetic and environmental responses to temperature of Drosophila melanogaster from a latitudinal cline. Genetics 146: 881-890.

Bettencourt BR, Kim IY, Hoffmann AA, Feder ME (2002). Response to natural and laboratory selection at the Drosophila HSP70 genes. Evolution 56: 1796-1801.

Calboli FCF, Kennington WJ, Patridge L (2003). QTL mapping reveals a striking coincidence in the positions of genomic regions associated with adaptive variation in body size in parallel clines of Drosophila melanogaster on different continents. Evolution 57: 2653-2658.

Chen B, Shilova VY, Zatsepina OG, Evgen'ev MB, Feder ME (2008). Location of $P$ element insertions in the proximal promoter region of $H s p 70 A$ is consequential for gene expression and correlated with fecundity in Drosophila melanogaster. Cell Stress Chaperones 13: 11-17.

Cossins AR, Bowler K (1987). Temperature Biology of Animals. Chapman and Hall: New York.

Ekengren S, Hulmark D (2001). A family of Turandot-related genes in humoral stress response of Drosophila. Biochem Biophys Res Commun 284: 998-1003.

Gockel J, Kennington WJ, Hoffmann A, Goldstein DB, Partridge L (2001). Nonclinality of molecular variation implicates selection in maintaining a morphological cline of Drosophila melanogaster. Genetics 158: 319-323.

Gong WJ, Golic KG (2006). Loss of HSP70 in Drosophila is pleitrophic, with effects on thermotolerance, recovery from heat shock and neurodegeneartaion. Genetics 172: 275-286.

Guerra D, Cavicchi S, Krebs RA, Loeschcke V (1997). Resistance to heat and cold stress in Drosophila melanogaster - intra and inter population variation in relation to climate. Genet Sel Evol 29: 497-510. 
Hedrick P (2000). Genetics of Populations. Jones and Bartlett Publishers: USA.

Hoffmann AA, Anderson A, Hallas R (2002). Opposing clines for high and low temperature resistance in Drosophila melanogaster. Ecol Lett 5: 614-618.

Hoffmann AA, Parsons PA (1991). Evolutionary Genetics and Environmental Stress. Oxford University Press: Oxford.

Hoffmann AA, Sorensen JG, Loeschcke V (2003). Adaptation of Drosophila to temperature extremes: bringing together quantitative and molecular approaches. J Therm Biol 28: 175-216.

Hogan NC, Slot F, Traverse KL, Garbe JC, Bendena WG, Pardue ML (1995). Stability of tandem repeats in Drosophila melanogaster Hsr-omega nuclear RNA. Genetics 139: 1611-1621.

Kennington WJ, Partridge L, Hoffmann AA (2006). Patterns of diversity and linkage disequilibrium within the cosmopolitan inversion $\operatorname{In}(3 R) P$ in Drosophila melanogaster are indicative of coadaptation. Genetics 172: 1655-1663.

Kimura MT, Ohtsu T, Yoshida T, Awasaki T, Lin FJ (1994). Climatic adaptations and distributions in the Drosophila takahashii species subgroup (Diptera: Drosopilaidae). J Nat Hist 28: 401-409.

Lakhotia SC (2003). The noncoding developmentally active and stress inducible hsr $\omega$ gene of Drosophila melanogaster integrates post-transcriptional processing of other nuclear transcripts. In: J Barciszewski and VA Erdmann (eds). Noncoding RNAs: Molecular Biology and Molecular Medicine, @ 2003 Eurekah.com, Kluwer Academic/Plenum Publishers, New York, Ch 14, pp 203-220.

Lakhotia SC, Singh AK (1985). Non-inducibility of the 93D puff in cold-reared larvae of Drosophila melanogaster. Chromosoma 92: 48-54.

Leemans R, Egger B, Loop T, Kammermeier L, Hartmann B, Certa $\mathrm{U}$ et al. (2000). Quantitative transcript imaging in normal and heat-shocked Drosophila embryos by using high-density oligonucleotide arrays. Proc Natl Acad Sci USA 97: 12138-12143.

Lindquist S (1980). Varying patterns of protein synthesis in Drosophila during heat shock: implications for regulation. Dev Biol 77: 463-479.

Lovejoy TE, Hanna L (eds.) (2006). Climate Change and Biodiversity. Yale University Press: New Haven, CT.

McColl G, Hoffmann AA, McKechnie SW (1996). Response of two heat shock genes to selection for knockdown heat resistance in Drosophila melanogaster. Genetics 143: 1615-1627.

McKechnie SW, Halford MM, McColl G, Hoffmann AA (1998). Both allelic variation and expression of nuclear and cytoplasmic transcripts of hsr-omega are closely associated with thermal phenotype in Drosophila. Proc Natl Acad Sci USA 95: 2423-2428.

Morgan TJ, Mackay TFC (2006). Quantitative trait loci for thermotolerance phenotypes in Drosophila melanogaster. Heredity 96: 232-242.

Norry FM, Dahlgaard J, Loeschcke V (2004). Quantitative trait loci affecting knockdown resistance to high temperature in Drosophila melanogaster. Mol Ecol 13: 3585-3594.
Pardue ML, Bendena WG, Fini ME, Garbe JC, Hogan NC, Traverse KL (1990). Hsr-omega, a novel gene encoded by a Drosophila heat shock puff. Biol Bull 179: 77-86.

Qin W, Neal SJ, Robertson RM, Westwood JT, Walker V (2005). Cold hardening and transcriptional change in Drosophila melanogaster. Insect Mol Biol 14: 607-613.

Rako L, Anderson AR, Sgrò CM, Stocker AJ, Hoffmann AA (2006). The association between the Inversion(3R)Payne and clinally varying traits in Drosophila melanogaster. Genetica 128: 373-384.

Rako L, Blacket MJ, McKechnie SW, Hoffmann AA (2007). Candidate genes and thermal phenotypes: identifying ecologically important genetic variation for thermotolerance in the Australian Drosophila melanogaster cline. Mol Ecol 16: 2948-2957.

Raymond M, Rousset F (1995). Genepop (version 1.2): population genetics software for exact tests and ecumenicism. J Hered 86: 248-249.

Rice WR (1989). Analyzing tables of statistical tests. Evolution 43: 223-225.

Sambrook J, Fritsch EF, Maniatis T (1989). Molecular Cloning a Laboratory Manual. Cold Spring Harbor Laboratory Press: USA.

Singh AK, Lakhotia SC (1984). Lack of effects of microtubule poisons on the 93D or 93D-like heat shock puffs in Drosophila. Ind J Exp Biol 22: 569-576.

Sokal RR, Rohlf FJ (1996). Biometry, 3rd edn. Freeman: New York.

Sørensen JG, Nielsen MM, Kruhøffer M, Justesen J, Loeschcke V (2005). Full genome gene expression analysis of the heat stress response in Drosophila melanogaster. Cell Stress Chaperones 10: 312-328.

Walldorf U, Richter S, Ryseck R-P, Steller JE, Bautz EKF, Hovemann B (1984). Cloning of heat-shock locus 93D from Drosophila melanogaster. EMBO J 3: 2499-2504.

Walser JC, Chen B, Feder ME (2006). Targets for evolution by $P$ transposable elements in Drosophila. PloS Genet 2: 1541-1555.

Weeks AR, McKechnie SW, Hoffmann AA (2002). Dissecting adaptive clinal variation: markers, inversions and size/stress associations in Drosophila melanogaster from a central field population. Ecol Lett 5: 756-763.

Zatsepina OG, Ulmasov KA, Beresten SF, Molodtsov B, Rybtsov SA, Evgenev MB (2000). Thermotolerant desert lizards characteristically differ in terms of heat-shock system regulation. J Exp Biol 203: 1017-1025.

Zatsepina OG, Velikodvorskaia VV, Molodtsov VB, Garbuz D, Lerman DN, Bettencourt BR et al. (2001). A Drosophila melanogaster strain from sub-equatorial Africa has exceptional thermotolerance but decreased Hsp70 expression. J Exp Biol 204: 1869-1881.

Zu K, Sikes ML, Beyer AL (1998). Separate roles in vivo for the two RNA binding domains of a Drosophila A1-hnRNP homolog. RNA 4: 1585-1598. 